\title{
Modelo de previsão da área de desgaste em pavimentos rodoviários com revestimento em tratamento superficial
}

\author{
Sérgio Pacífico Soncim, José Leomar Fernandes Júnior², Luis Edmundo Prado de Campos³
}

\begin{abstract}
Resumo: Este trabalho apresenta o desenvolvimento de um modelo de previsão da área de desgaste para a malha rodoviária, construída em tratamento superficial duplo, do Estado da Bahia. Teve como ponto de partida um planejamento fatorial, elaborado com base em informações de um banco de dados de rodovias, fornecido pelo Departamento de Infraestrutura de Transportes da Bahia (DERBA). Os fatores considerados foram a idade do pavimento, o tráfego e a pluviometria. A ANOVA foi a técnica utilizada para avaliar a significância dos fatores e definir os parâmetros do modelo de desempenho. Para a verificação da adequação do modelo estatístico utilizou-se a reta de ajuste dos dados previstos aos observados, com determinação do coeficiente de correlação $(r)$, a análise de resíduos, e o gráfico de probabilidade normal dos resíduos. O modelo desenvolvido neste trabalho apresentou ajuste aos dados observados e coeficiente de correlação $r=0,74$.
\end{abstract}

Palavras-chave: Pavimentos asfálticos. Sistemas de gerência. Modelos de desempenho. Desgaste.

Abstract: This paper presents the development of a raveling prediction model for the highway network built in double surface treatmen in State of Bahia. Its starting point was a factorial design, based on information from a highway database provided by the Departamento de Infraestrutura de Transportes da Bahia (DERBA). The factors considered were the pavement age, traffic and rainfall. The Analysis of Variance was the technique used to analyze and assess the significant factors and define the parameters of the performance model. To verify the adequacy of the statistical model was used the straight fit to the observed data set, with determination of the correlation coefficient $(r)$, residue analysis, and normal probability plot of the residuals. The model developed in this work showed fit to the data and a correlation coefficient $r=0.74$.

Keywords: Asphalt pavements. Management systems. Performance models. Raveling.

\section{INTRODUÇÃO}

Um Sistema de Gerência de Pavimentos (SGP) é um conjunto de métodos que auxilia os tomadores de decisões na busca por melhores estratégias para fornecer e manter pavimentos em condições adequadas de serventia por um determinado período de tempo. Sua função é melhorar a eficiência das decisões e ampliar suas possibilidades, avaliar as consequências das decisões tomadas, facilitar a coordenação de atividades dentro de um órgão rodoviário e assegurar a consistência das decisões tomadas em diferentes níveis de gerência dentro da mesma organização.

Modelos de previsão da condição do pavimento fazem parte de um SGP e são utilizados tanto em nível de rede, para planejamento, estimativa das necessidades totais de manutenção e reabilitação, priorização de projetos e programação de investimentos, como em nível de projeto,

\footnotetext{
1 Universidade Federal de Itajubá (sergiops@unifei.edu.br)

2 Universidade de São Paulo (leomar@sc.usp.br).

3 Universidade Federal da Bahia (ledmundo@ufba.br).

Manuscrito recebido em 07/02/2014 e aprovado para publicação em 04/05/2014. Este artigo é parte de TRANSPORTES v. 22, n. 2, 2014. ISSN: 2237-1346 (online).
}

DOI: http://dx.doi.orq/10.14295/transportes.v22i2.763. para a definição de atividades de manutenção e de reabilitação. Falhas no processo de previsão da condição futura do pavimento podem resultar em escolhas de estratégias erradas e, consequentemente, em utilização ineficiente de recursos.

Os modelos de previsão de desempenho devem refletir as condições às quais eles são aplicados e devem ser desenvolvidos a partir de dados locais. O Brasil representa um exemplo excelente dessa necessidade, pois possui condições climáticas diversificadas e grande variedade de solos (Queiroz, 1984). Os modelos de desempenho devem retratar, da melhor forma possível, as condições regionais, uma vez que cada região apresenta características diferenciadas como tráfego, clima, capacidade de suporte do subleito, tipo de materiais empregados na construção, além de diferentes técnicas e controles construtivos.

O objetivo deste trabalho foi desenvolver um modelo de previsão da área de desgaste para a rede rodoviária em tratamento superficial duplo do estado da Bahia. Os fatores considerados para o desenvolvimento do modelo foram a idade do pavimento, o tráfego e a pluviometria. Foram utilizadas informações de um banco de dados de levantamentos de rodovias do Depar- 
tamento de Infraestrutura de Transportes do Estado da Bahia - DERBA. ANOVA foi a técnica utilizada para avaliar a significância dos fatores e definir os parâmetros do modelo de desempenho. Para a verificação da adequação do modelo estatístico utilizou-se a reta de ajuste dos dados previstos aos observados, com determinação do coeficiente de correlação (r), a análise de resíduos, e o gráfico de probabilidade normal dos resíduos.

\section{MODELOS DE DESEMPENHO DE PAVIMENTOS ASFÁLTICOS}

Desde que os conceitos de gerência de pavimentos foram iniciados na década de 60 , muitos modelos de previsão de desempenho foram desenvolvidos como forma de tentar inferir sobre o futuro desempenho dos pavimentos, com propósitos de planejamento. Haas, Hudson e Zaniewski (1994) agrupam esses modelos em quatro tipos básicos:

- Modelos Mecanísticos: esses modelos excluem todas as inferências empíricas na estimativa da deterioração do pavimento. Todas as respostas e seus efeitos na estrutura do pavimento são puramente mecanísticos e baseados na representação física do processo de deterioração, o qual é representado por parâmetros de resposta como tensões, deformações e deflexões.

- Modelos empírico-mecanísticos: nesses modelos as respostas estruturais (tensões, deformações e deslocamentos) são correlacionadas com dados experimentais sobre a evolução da deterioração estrutural ou funcional dos pavimentos, por meio de análise de regressão.

- Modelos Empíricos: Modelos de previsão empíricos relacionam um determinado índice de desempenho do pavimento (variável dependente), como por exemplo, indicadores subjetivos (ISA - Índice de Serventia Atual, por exemplo) ou objetivos (acúmulo de deformação permanente nas trilhas de roda, por exemplo) a variáveis independentes, como as solicitações do tráfego, geralmente representadas pelo número de aplicações equivalentes do eixo-padrão, ou os efeitos do meio ambiente (temperatura e teor de umidade), geralmente representados pelo número de anos, que quantificam os ciclos climáticos.

- Modelos subjetivos: são desenvolvidos com base na experiência e no conhecimento adquirido pelos especialistas responsáveis pela ge- rência de uma determinada rede. Os modelos são desenvolvidos com o auxílio de técnicas que auxiliam na "captura" da experiência de forma estruturada.

\section{Modelos de desempenho desenvolvidos para redes de rodovias no Brasil}

Desde a década de 70 estudos têm sido realizados no Brasil com o objetivo de desenvolvimento de modelos de desempenho para utilização em gerência de pavimentos. Merecem destaque os estudos iniciados em 1975 pela Empresa Brasileira de Planejamento de Transportes (GEIPOT, 1981) que resultaram na Pesquisa de Inter-Relacionamento de Custos de Construção, Conservação e Utilização de Rodovias (PICR). Nesta pesquisa, Queiroz (1981) utilizou dados obtidos da observação de 63 seções de pavimento em serviço, da malha rodoviária compreendida no quadrilátero Brasília, Goiânia, Belo Horizonte e São Paulo, para o desenvolvimento de modelos de desempenho. Alguns dos principais modelos resultantes da pesquisa foram apresentados anteriormente.

Paterson (1987) desenvolveu as equações de desempenho de pavimentos utilizadas no The Highway Design and Maintenance Standards Model (Watanada et al., 1987). A base empírica para o desenvolvimento dos modelos de regressão foi a mesma utilizada por Queiroz (1981) durante a PICR. O motivo da escolha foi a grande quantidade de dados observados ao longo do tempo que incluem irregularidades, trincamento, desgaste, deformações permanentes, atividades de manutenção, tráfego e pluviometria para uma programação fatorial de trechos de pavimentos em serviço (Paterson, 1989).

Marcon (1996) desenvolveu modelos de previsão de desempenho com base em dados da rede rodoviária do estado de Santa Catarina. Os dados foram coletados em 1990 e referiam-se a deflexões, irregularidades de superfície, defeitos, materiais empregados, espessuras das camadas e tráfego do estado, dividido em três regiões. O desempenho dos pavimentos foi representado pelas variáveis dependentes: quociente de irregularidade, deflexões, índice de gravidade global, trincamento total e afundamento nas trilhas de roda, tendo como variável independente o intervalo de tempo, em anos, ou o número de operações do eixo padrão de $80 \mathrm{kN}$. Com base nos dados, foram testados cinco tipos de equações (linear, logarítmica, polinomial, exponencial e 
potencial) por meio de regressão. Para cada par de variáveis foi indicada a equação que apresentava o melhor coeficiente de determinação $\left(\mathrm{R}^{2}\right)$.

Yshiba (2003) desenvolveu modelos estatísticos que representam os efeitos dos fatores idade, tráfego e número estrutural sobre o desempenho de pavimentos, quantificado em termos de irregularidade longitudinal e deflexões. A técnica usada foi a análise da variância (ANOVA) aplicada aos dados de levantamentos realizados em 1995 e 1998 na malha rodoviária do Estado do Paraná. A ANOVA permite a determinação do nível de significância de fatores pré-selecionados e de suas interações, bem como a modelagem do desempenho dos pavimentos. Yshiba (2003) desenvolveu, também, modelos de desempenho probabilísticos para cada uma das células das matrizes fatoriais, que correspondem às combinações de fatores considerados para a análise da ANOVA, a partir de avaliações realizadas por engenheiros do DERPR e mediante o estabelecimento de matrizes de estabilidade de Markov.

Benevides (2006) desenvolveu modelos estatísticos baseado em levantamentos realizados em 72 trechos da malha rodoviária pavimentada da região metropolitana de Fortaleza. Nos modelos estatísticos foram estabelecidas equações de regressão, utilizando a ANOVA. Foram determinadas equações de evolução para os defeitos/severidades efetuados por meio de Levantamento Visual Contínuo (LVC) em 2004 e 2005, assim como equações de regressão que relacionam os valores de irregularidade longitudinal obtido com os perfilômetros inercial e a laser. Também foram estabelecidas equações relacionando a irregularidade longitudinal com os defeitos indicados pelo LVC, com os valores do Índice de Condição do Pavimento (ICP) dos modelos probabilísticos, com a deflexão e com o número estrutural corrigido. Modelos de regressão múltipla e evolução de irregularidade com o ICP, a deflexão e os defeito/severidade foram também estabelecidos. Benevides (2006) também desenvolveu modelos probabilísticos. Foram estabelecidos vetores estados inicial dos trechos, considerando a situação atual do pavimento, e utilizadas as matrizes de probabilidade de transição de Markov para gerar os modelos probabilísticos e assim determinar o ICP.

Albuquerque et al. (2007) desenvolveram modelos de Previsão de Desempenho a partir de dados de levantamentos de deflexão com FWD
(Falling Weight Deflectometer), de deflexão com Viga Benkelman e de irregularidade longitudinal para pavimentos em tratamento superficial dos Estados do Ceará e Paraíba. O clima foi considerado pelo Índice de Aridez desenvolvido pela UNESCO e a capacidade estrutural do pavimento foi expressa pelo número estrutural corrigido (SNC). Já a idade do pavimento e as cargas de tráfego foram expressas em termos de número acumulado de repetições do eixo padrão. Todos os modelos foram construídos através de regressão por crescimento exponencial.

Soncim e Fernandes Jr. (2012) desenvolveram um modelo de previsão de irregularidade longitudinal para a malha rodoviária, em tratamento superficial duplo, do estado da Bahia. Teve como ponto de partida um planejamento fatorial, elaborado com base em informações de um banco de dados de rodovias, fornecido pelo Departamento de Infraestrutura de Transportes da Bahia (DERBA). Os dados de irregularidade longitudinal foram coletados em 2009, quando foram percorridos aproximadamente $3.000 \mathrm{~km}$ de rodovias e executados $650 \mathrm{~km}$ de levantamentos. Os fatores considerados foram a idade do pavimento, o tráfego e a pluviometria. A ANOVA foi o método de análise estatística dos dados utilizado para avaliar a significância dos fatores e definir os parâmetros do modelo de desempenho.

\section{ESCOLHA DAS VARIÁVEIS DO MODELO}

A escolha das variáveis deve ser relacionada ao nível de gerência com que se pretende desenvolver o estudo. Neste trabalho, as variáveis foram escolhidas com base na disponibilidade de informações no banco de dados para o desenvolvimento de um modelo de desempenho para aplicação em gerência em nível de rede, mas também foram levados em consideração os principais estudos desenvolvidos para as condições nacionais, conforme apresentado na revisão bibliográfica.

Foram considerados critérios apresentados por Bennett e Paterson (2000), que permitem estruturar o gerenciamento de informações de forma que sejam adequadas às necessidades, para os diferentes níveis de gerência de pavimentos. Também foram observadas as recomendações do Standard Guide for Prioritization of Data Needs for Pavement Management (ASTM 
E - 96, Revisada 2002). Bennet e Paterson (2000) apresentam os IQL (Information Quality Levels) em cinco níveis, variando de 1 a $5 \mathrm{em}$ função dos objetivos do nível de gerência, como apresentado a seguir:

- IQL1 e IQL2 - representam os níveis de detalhamento de informações típico da gerência em nível de projeto, onde muitos atributos devem ser medidos e coletados. Esses níveis são indicados para as agências que gerenciam vias expressas ou rodovias principais e são dotadas de orçamento disponível para suas pesquisas.

- IQL3 - exige menor detalhamento, geralmente 2 ou 3 atributos, os quais podem ser usados em gerência em nível de rede. Indicado para o gerenciamento de rodovias rurais ou locais, sendo simples, mas eficaz para o seu propósito.

- IQL4 e IQL5 - representam os níveis de informação que são usados em nível de planejamento. São indicados para o nível de gerência onde as estatísticas rodoviárias e os indicadores de desempenho são direcionados para os gerenciadores e ao público, pois são de fácil entendimento, sem a necessidade de conhecimento aprofundado sobre o assunto.

O Standard Guide for Prioritization of Data Needs for Pavement Management (ASTM
E - 96, Revisada 2002) estabelece critérios baseados no nível de gerência que se deseja (rede ou projeto) e na classificação funcional das rodovias, conforme apresentado na Tabela 1. Do mesmo modo, as rodovias são subdivididas em rodovias com alto volume de tráfego, para um Volume Médio Diário Anual (VMDA) superior a 10.000 , e rodovias com baixo volume de tráfego, para rodovias com VMDA inferior a 10.000. A importância relativa dos dados necessários à gerência, em função dos critérios citados, é, então, classificada como alta (A), média (M) e baixa (B). Percebe-se que a importância dos dados cresce em função do nível de gerência (de rede para projeto), da classificação da via e do tráfego que a mesma suporta.

Com base nos dados disponíveis no banco de dados e nos conceitos de gerência em nível de rede, foram selecionadas as seguintes variáveis independentes para desenvolvimento do modelo de desempenho para da área de desgaste para pavimentos em tratamento superficial duplo: tráfego (TA) desde o início da operação do trecho (representado pelo número equivalente de operações do eixo padrão de $80 \mathrm{kN}$ ); idade do pavimento (ID, em anos), e pluviometria (PL, em mm anuais).

Tabela 1 - Nível de importância dos dados necessários à gerência de pavimentos - adaptada ASTM E - 96 ( 2002)

\begin{tabular}{|c|c|c|c|c|c|c|c|c|}
\hline \multirow{4}{*}{ Tipos de dados } & \multicolumn{4}{|c|}{ Nível de Rede } & \multicolumn{4}{|c|}{ Nível de Projeto } \\
\hline & \multicolumn{2}{|c|}{ Principais } & \multicolumn{2}{|c|}{ Secundárias } & \multicolumn{2}{|c|}{ Principais } & \multicolumn{2}{|c|}{ Secundárias } \\
\hline & $\begin{array}{c}\text { Tráfego } \\
\text { Alto }\end{array}$ & $\begin{array}{c}\text { Tráfego } \\
\text { Baixo }\end{array}$ & $\begin{array}{c}\text { Tráfego } \\
\text { Alto }\end{array}$ & $\begin{array}{c}\text { Tráfego } \\
\text { Baixo }\end{array}$ & $\begin{array}{c}\text { Tráfego } \\
\text { Alto }\end{array}$ & $\begin{array}{c}\text { Tráfego } \\
\text { Baixo }\end{array}$ & $\begin{array}{c}\text { Tráfego } \\
\text { Alto }\end{array}$ & $\begin{array}{c}\text { Tráfego } \\
\text { Baixo }\end{array}$ \\
\hline & \multicolumn{8}{|c|}{ Dados Relacionados ao Desempenho } \\
\hline Irregularidade Long. & A & A & $\mathrm{M}$ & $\mathrm{M}$ & $\mathrm{A}$ & $\mathrm{A}$ & A & A \\
\hline Defeitos de superfície & A & A & A & A & A & A & A & A \\
\hline Aderência pneu-pavimento & $\mathrm{M}$ & $\mathrm{M}$ & $\mathrm{M}$ & $\mathrm{B}$ & A & B & A & B \\
\hline Deflexões recuperáveis & $\mathrm{M}$ & $\mathrm{B}$ & $\mathrm{M}$ & B & A & A & A & $\mathrm{M}$ \\
\hline \multirow[t]{2}{*}{ Materiais } & $\mathrm{B}$ & $\mathrm{B}$ & $\mathrm{B}$ & B & A & $\mathrm{M}$ & $\mathrm{M}$ & B \\
\hline & \multicolumn{8}{|c|}{ Dados Relacionados ao Histórico } \\
\hline Construção & A & A & $\mathrm{M}$ & B & A & A & $\mathrm{M}$ & B \\
\hline Manutenção & A & $\mathrm{M}$ & $\mathrm{M}$ & $\mathrm{B}$ & A & $\mathrm{M}$ & $\mathrm{M}$ & $\mathrm{B}$ \\
\hline Tráfego & A & $\mathrm{M}$ & $\mathrm{M}$ & B & A & $\mathrm{M}$ & $\mathrm{M}$ & B \\
\hline \multirow[t]{2}{*}{ Acidentes } & A & $\mathrm{M}$ & $A$ & $\mathrm{M}$ & A & A & A & $\mathrm{M}$ \\
\hline & \multicolumn{8}{|c|}{ Dados Relacionados à Geometria } \\
\hline Larguras de pista & A & A & A & A & A & A & A & A \\
\hline Curvatura horizontal & A & $\mathrm{M}$ & $\mathrm{M}$ & B & A & $\mathrm{M}$ & $\mathrm{M}$ & $\mathrm{B}$ \\
\hline Subidas / descidas & $\mathrm{M}$ & $\mathrm{B}$ & $\mathrm{M}$ & B & $A$ & $\mathrm{M}$ & $\mathrm{M}$ & $\mathrm{M}$ \\
\hline Curvatura vertical & $\mathrm{M}$ & $\mathrm{B}$ & B & B & $\mathrm{M}$ & B & $\mathrm{B}$ & B \\
\hline \multirow[t]{2}{*}{ Acostamento } & $A$ & $\mathrm{M}$ & $A$ & $\mathrm{M}$ & $A$ & $A$ & $A$ & $\mathrm{M}$ \\
\hline & \multicolumn{8}{|c|}{ Dados Relacionados ao Meio Ambiente } \\
\hline Drenagem & A & $\mathrm{M}$ & A & $\mathrm{M}$ & A & $\mathrm{M}$ & A & $\mathrm{M}$ \\
\hline Clima & A & $\mathrm{M}$ & $\mathrm{M}$ & $\mathrm{B}$ & A & $\mathrm{M}$ & $\mathrm{M}$ & $\mathrm{B}$ \\
\hline
\end{tabular}


Idade da Construção (anos)

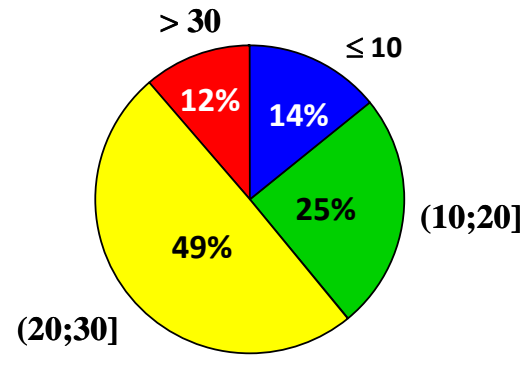

(a)

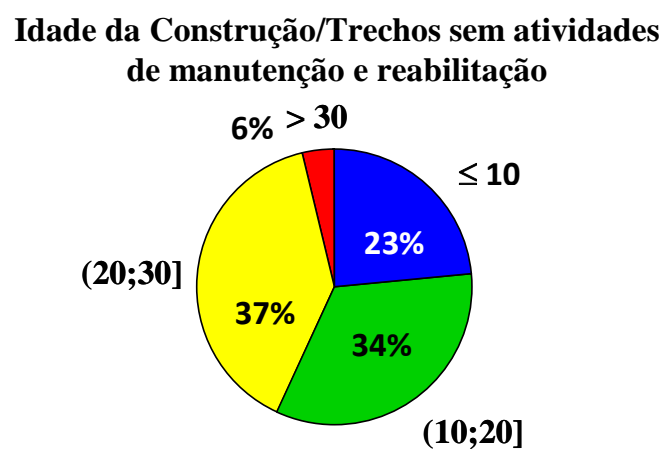

(b)

Figura 1 - Idade da construção (a) e idade da construção (anos) para os trechos sem atividades de manutenção e reabilitação (b)

\section{BANCO DE DADOS DA REDE RODOVIÁRIA DO ESTADO DA BAHIA}

O banco de dados utilizado nesta pesquisa faz parte dos estudos desenvolvidos pelo DERBA (2005), para a implementação do programa de restauração e manutenção rodoviária. Para a sua elaboração foram coletados dados sobre as condições de superfície dos pavimentos de aproximadamente $9.000 \mathrm{~km}$ de rodovias $(745$ trechos) durante o ano de 2004. Além disso, estão incluídas informações cadastrais como o tipo de revestimento existente, tenham eles sofrido alguma atividade de manutenção e reabilitação ou não, informações referentes à geometria, ao relevo, à idade dos pavimentos, à pluviometria, às condições de superfície e ao tráfego.

\section{Idade dos pavimentos}

O banco de dados de rodovias do estado da Bahia é composto por rodovias com idade superior a 20 anos, em sua maioria, conforme pode ser observado na Figura 1 (a). Apenas uma pequena parte das rodovias (14\%) apresenta idade inferior a 10 anos. A Figura 1(b) representa o quantitativo de rodovias que não sofreram atividades de manutenção e reabilitação até a data da realização da coleta de dados pelo DERBA, no ano de 2004. Pode-se observar que aproximadamente $43 \%$ desses trechos apresentam idade superior a 20 anos e aproximadamente $23 \%$ apresentam idade inferior a 10 anos.

\section{Pluviometria}

O gráfico da Figura 2 mostra a distribuição de chuvas para os trechos rodoviários disponíveis no banco de dados. Pode-se observar que $40 \%$ dos trechos apresentam pluviometria menor ou igual a $500 \mathrm{~mm} / \mathrm{ano}$ e $25 \%$ dos trechos apresentam regime pluviométrico entre 500 e $800 \mathrm{~mm}$, ou seja, assemelham-se ao regime pluviométrico típico da maior parte do estado da Bahia, característicos das regiões de clima árido a semiúmido, de acordo com a classificação climática de Thornthwaite.

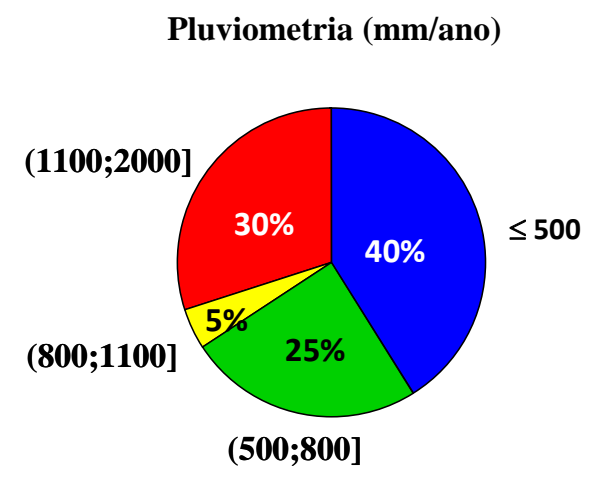

Figura 2 - Distribuição de chuvas para os trechos rodoviários disponíveis no banco de dados 
Tipos de revestimentos asfálticos

A Figura 3 (a) mostra a distribuição, como construído, dos principais tipos de revestimento asfálticos. Pode-se perceber a predominância de revestimentos do tipo tratamento superficial simples (TSS) e duplo (TSD) com aproximadamente $50 \%$ do total, seguido do tratamento contra/pó (TCP), com $17 \%$ do total. A Figura 3 (b) apresenta os revestimentos contidos no levantamento e que não haviam sofrido algum tipo de atividade de manutenção e reabilitação até 2004.
Pode-se perceber que os trechos em areia asfalto usinado a quente (AAUQ) e tratamento contra/pó sofreram intervenções e foram substituídos por outros tipos de revestimentos. Os tratamentos superficiais representam $67 \%$ do total dos trechos, seguidos pelos trechos em concreto betuminoso usinado a quente (CBUQ), com $16 \%$ do total. As lamas asfálticas (LA) e outros (O) pavimentos representam uma porcentagem inferior a 3\% do total.

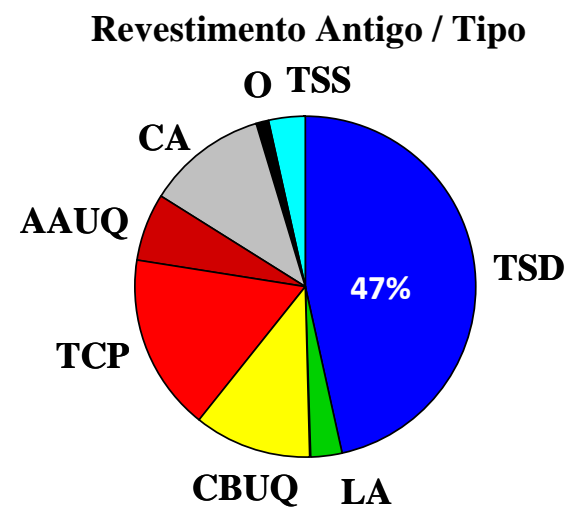

(a)

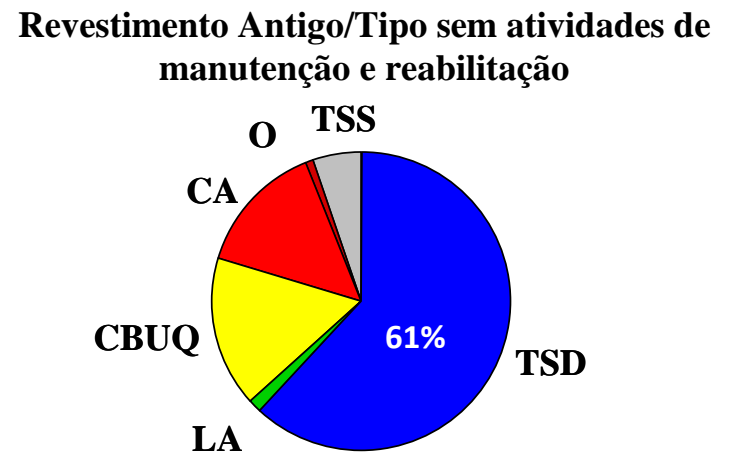

(b)

Figura 3 - Principais tipos de revestimento asfálticos como construído (a); Pavimentos que não haviam sofrido atividades de manutenção e reabilitação até o ano de 2004 (b)

Os pavimentos antigos, que sofreram atividades de manutenção e reabilitação, foram substituídos principalmente por tratamentos superficiais, em sua maioria, seguidos por CBUQ, como pode ser observado na Figura 4.

\section{Tráfego}

As informações sobre as características dos veículos que trafegam na rede rodoviária do Estado da Bahia, com relação a tipos e volumes médios diários, foram obtidas por meio de contagens volumétricas e classificatórias em 50 postos, realizadas pelo DERBA em 2004. As informações sobre carregamento dos veículos, como estimativa da quantidade de veículos carregados, veículos vazios e veículos infratores (veículos com excesso de PBT e/ou excesso por eixo) foram fornecidas pelo DERBA, referente a pesagens realizadas em 2005 e 2006 em 6 postos de pesagem portátil dinâmica de veículos de carga em rodovias estaduais e federais no estado da Bahia. A Figura 5 mostra a distribuição do VMDA para os 745 trechos presentes no banco de dados. Pela análise da figura pode-se notar que mais de $50 \%$ dos trechos pesquisados apre- sentam VMDA menor ou igual a 1000 veículos por dia. Apenas uma pequena proporção apresenta VMDA superior a 4000 veículos por dia.

\section{PLANEJAMENTO FATORIAL}

O planejamento fatorial do experimento considerou os fatores idade do pavimento (ID), tráfego, representado pelo número de aplicações equivalentes do eixo-padrão desde a sua abertura até o ano de 2004 (TA), e pluviometria (PL). Foram adotados dois níveis para cada fator, obtendo-se um experimento fatorial tipo $2^{3}=8$. Houve réplicas, ou seja, repetições de avaliações em seções de pavimento pertencentes à uma mesma célula de uma matriz fatorial, cujos resultados foram obtidos com os mesmos equipamentos, equipe de campo, procedimentos e espaço de inferência.

Os níveis foram escolhidos de acordo com a distribuição dos fatores idade do pavimento (ID), tráfego desde a abertura do pavimento até o ano de 2004 (TA) e pluviometria (PL), para os trechos em tratamento superficial duplo que não haviam sofrido atividades de manutenção e rea- 
bilitação até o ano de 2004, de acordo com o banco de dados do DERBA, resultando num total de 295 trechos. A Tabela 2 apresenta, para os

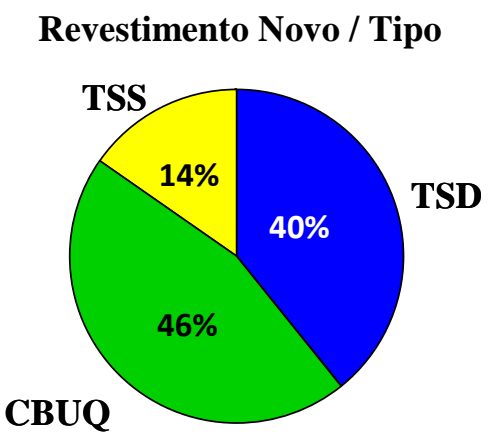

Figura 4 - Principais tipos de revestimentos utilizados em substituição aos AAUQ e aos tratamentos contra/pó
295 trechos, os valores médios, mínimos e máximos para os fatores ID, TA e PL.

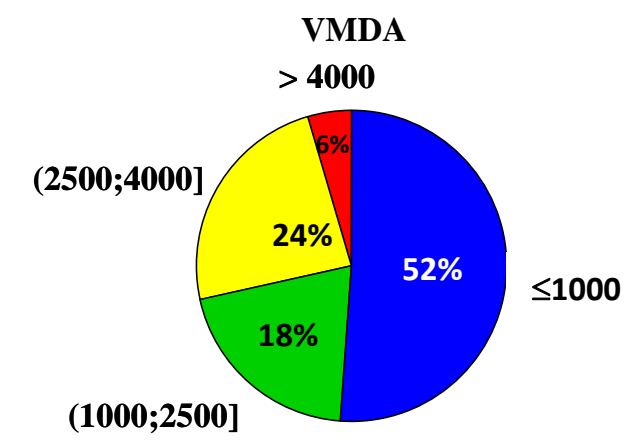

Figura 5 - Contagens volumétricas para os trechos do banco de dados

\begin{tabular}{|c|c|c|c|}
\hline $\begin{array}{l}\text { Fator } \\
\text { ID }\end{array}$ & $\begin{array}{c}\text { Média } \\
19\end{array}$ & $\begin{array}{c}\text { Mínimo } \\
6\end{array}$ & $\begin{array}{c}\text { Máximo } \\
36\end{array}$ \\
\hline TA & 994303 & 85000 & 4171043 \\
\hline$P L$ & 523 & 303 & 1930 \\
\hline
\end{tabular}

Alguns trechos não tinham informações sobre o valor da variável dependente área de desgaste $\left(A_{\text {Desg }}\right)$ e portanto, não foram incluídos no planejamento fatorial, resultando em um total de 70 trechos utilizados. Os valores limites dos níveis dos fatores foram escolhidos de maneira que as células do arranjo do experimento fatorial fossem preenchidas com pelo menos duas réplicas. Os valores das réplicas utilizados para o desenvolvimento dos modelos foram escolhidos de forma aleatória e os demais valores foram utilizados na fase da validação dos modelos. O planejamento fatorial para os trechos em TSD sem atividades de manutenção e reabilitação até 2004 é apresentado seguir:

- Fator ID: idade do pavimento, com dois níveis, codificados por i1 $(-1)$ e i2 $(+1)$ :

nível id ${ }_{1}$ : idade do pavimento $\leq 15$ anos; nível $i_{2}$ : idade do pavimento $>15$ anos.

- Fator TA: tráfego desde a sua abertura até o ano de 2004, com dois níveis, codificados por ta $1(-1)$ e ta $2(+1)$ :

nível ta: tráfego $\leq 6 \times 10^{5}$ operações do eixo padrão de $80 \mathrm{kN}$;

nível ta2: tráfego $>6 \times 10^{5}$ operações do eixo padrão de $80 \mathrm{kN}$.
- Fator PL: pluviometria, com dois níveis, codificados por $\mathrm{pl}_{1}(-1)$ e $\mathrm{pl}_{2}(+1)$ :

nível $\mathrm{pl}_{1}$ : pluviometria $\leq 445 \mathrm{~mm} / \mathrm{ano}$; nível $\mathrm{pl}_{2}$ : pluviometria $>445 \mathrm{~mm} / \mathrm{ano}$.

\section{DESENVOLVIMENTO DO MODELO DE ÁREA DE DESGASTE}

A Análise de variância é um teste estatístico que visa verificar se existe uma diferença significativa entre as médias provenientes de amostras distintas e se os fatores exercem influência no modelo. A distribuição $F$ é utilizada pela Análise da Variância na decisão de aceitar ou rejeitar a hipótese de igualdade entre grupos de dados (hipótese nula). O objetivo do teste é obter o valor de $F_{\text {observado, dado pela relação entre }}$ a variância entre grupos e a variância dentro de grupos. Se o Fobservado for maior que o $F_{\text {crítico, }}$ para um dado nível de significância $\alpha$, então a hipótese nula é rejeitada. A Tabela 3 apresenta o resumo do cálculo da Análise de Variância dos fatores e suas interações, considerando-se a variável dependente irregularidade longitudinal, para um nível de significância de $5 \%(\alpha=0,05$, $\left.F_{\text {crítico }}=5,32\right)$. 


$\begin{array}{ccccccccc}\text { Fator } & \begin{array}{c}\text { Soma } \\ \text { Quadrática }\end{array} & \begin{array}{c}\text { Graus de } \\ \text { Liberdade }\end{array} & \begin{array}{c}\text { Média } \\ \text { Quadrática }\end{array} & \text { Efeito } & \text { Coeficiente } & \text { Fo } & \text { Significativo } & \begin{array}{c}\text { Valor } \\ \mathbf{P}\end{array} \\ \text { ID } & 2727,398 & 1 & 2727,398 & 26,112 & 13,056 & 25,594 & \text { SIM } & 0,001 \\ \text { TA } & 1500,013 & 1 & 1500,013 & 19,365 & 9,683 & 14,076 & \text { SIM } & 0,006 \\ \text { PL } & 125,956 & 1 & 125,956 & 5,612 & 2,806 & 1,182 & \text { NÃO } & 0,309 \\ \text { ID*TA } & 567,035 & 1 & 567,035 & 11,906 & 5,953 & 5,321 & \text { SIM } & 0,050 \\ \text { ID*PL } & 0,070 & 1 & 0,070 & -0,133 & -0,066 & 0,001 & \text { NÃO } & 0,980 \\ \text { TA*PL } & 53,451 & 1 & 53,451 & 3,656 & 1,828 & 0,502 & \text { NÃO } & 0,499 \\ \text { ID*TA*PL } & 177,036 & 1 & 177,036 & 6,653 & 3,326 & 1,661 & \text { NÃO } & 0,233 \\ \text { Erro } & 852,498 & 8 & 106,562 & & & & & \\ \text { Total } & 6003,457 & 15 & & & 31,415 & & & \end{array}$

A análise de regressão foi usada para a determinação dos parâmetros do modelo estatístico para previsão $\mathrm{Da}$ área de desgaste, conforme apresentado na Equação 1. As equações polino- miais correspondentes aos fatores idade do pavimento, tráfego e pluviometria são apresentadas nas Equações 2 e 3.

$$
\begin{gathered}
A_{\text {Desg }}=31,4+13,1 P(I D)+9,7 P(T A)+5,9 P(I D) P(T A) \\
R^{2}=0,80 \\
P(I D)=\frac{I D-21}{17} \\
P(T A)=\frac{T A-9,5 \times 10^{5}}{2,2 \times 10^{6}}
\end{gathered}
$$

Em que

$P(I D)$ : equação polinomial para o fator idade desde a construção; tráfego;

$P(T A)$ : equação polinomial para o fator

ID: valor quantitativo da variável Idade do pavimento, em anos;

TA: valor quantitativo da variável tráfego, em número de aplicações equivalentes do eixopadrão;

Para a verificação da adequação dos modelos estatísticos utilizou-se a reta de ajuste dos dados previstos aos observados, com determinação do coeficiente de correlação (r) correspondente (Figura 6), a análise de resíduos (Figura 7), e o gráfico de probabilidade normal dos resíduos (Figura 8). Deve-se destacar que o conjunto de dados observados, utilizados para validação do modelo, também foram coletados na etapa de levantamento de campo, em seções de pavimentos diferentes das que foram consideradas para o desenvolvimento do modelo de previsão de desempenho.

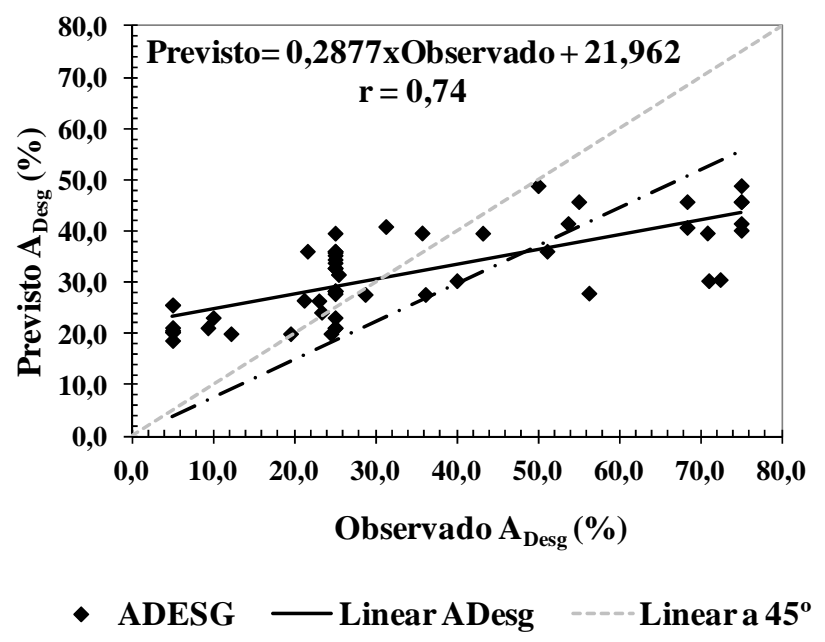

Figura 6 - Correlação dos valores observados com os valores previstos 


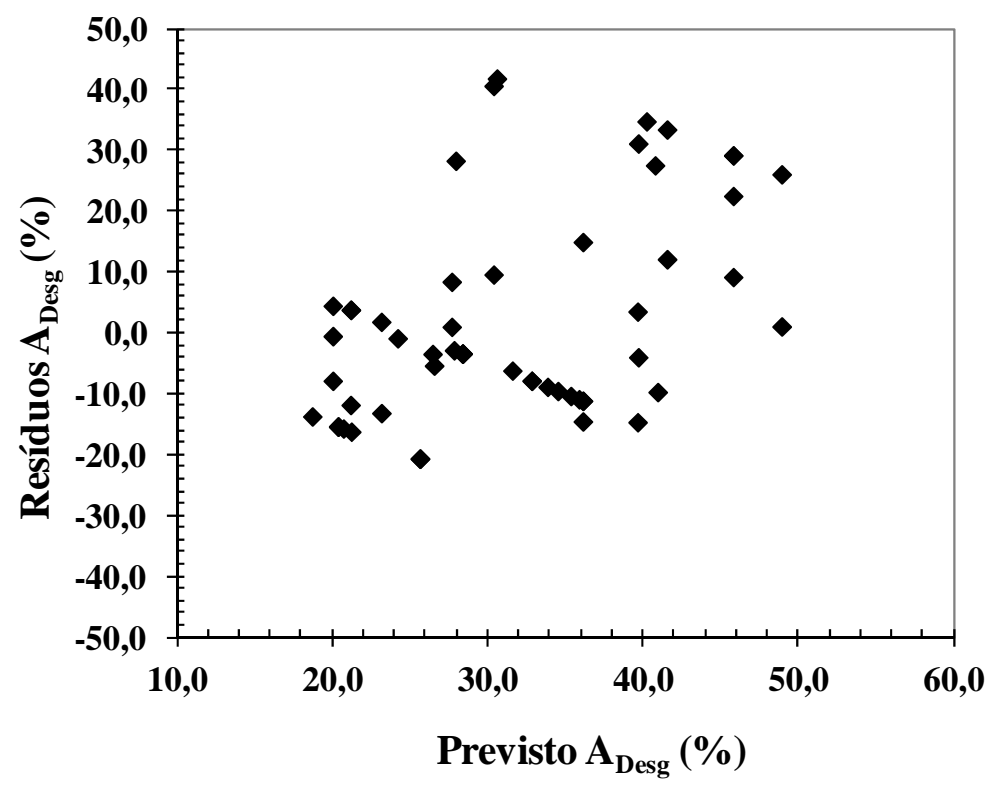

Figura 7 - Gráfico de resíduos do modelo obtido

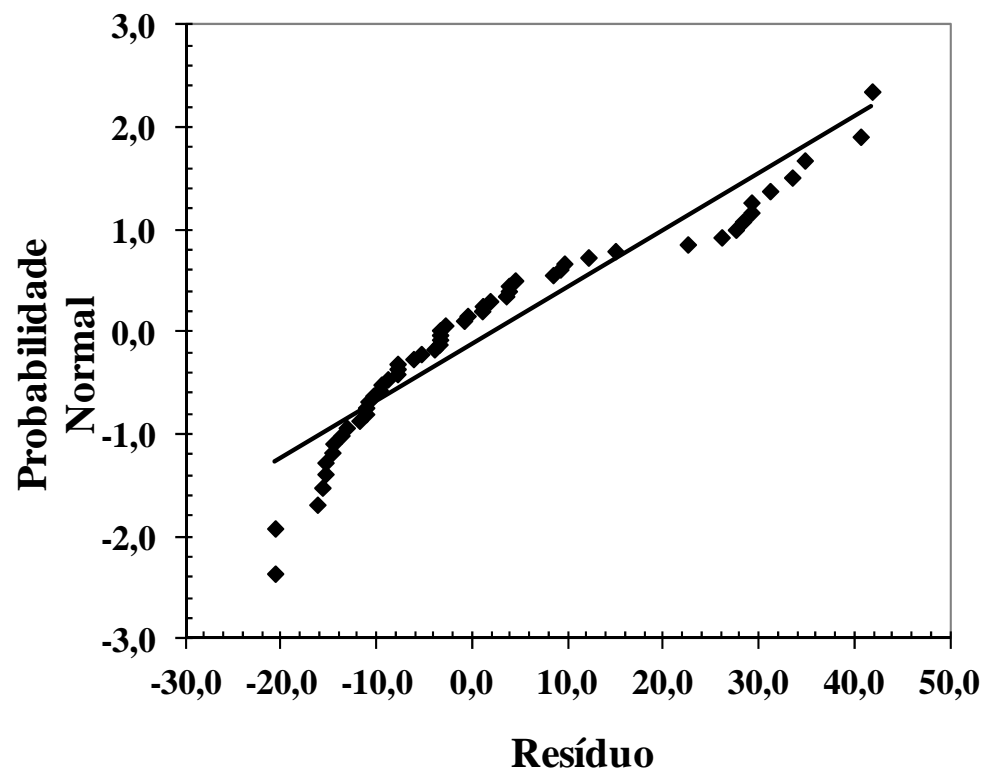

Figura 8 - Gráfico de distribuição de probabilidade normal dos resíduos

\section{ANÁLISE DOS EFEITOS DOS FATORES}

Os efeitos dos fatores foram avaliados em gráficos em três dimensões, pois a consideração de dois fatores implica no terceiro fator ser constante e igual a zero. Dessa forma é possível avaliar o comportamento de duas variáveis, de forma simultânea. As Figuras 9, 10 e 11 mostram os efeitos dos fatores idade, tráfego e pluviometria sobre a área de desgaste (ADesg), com base no modelo da Equação 1. Pela análise da Figura 9 pode-se observar que a ADesg aumenta quando os fatores idade e tráfego mudam do nível inferior (-1) para o nível superior $(+1)$, sendo o efeito da idade do pavimento maior do que o efeito do tráfego. Analisando-se a figura 10 pode-se observar que a ADesg aumenta quando a idade muda do nível inferior (-1) para o nível superior $(+1)$. De maneira semelhante, a Figura 11 mostra que a ADesg aumenta quando o tráfego muda do nível inferior (-1) para o nível superior $(+1)$. Entretanto, em ambos os casos, a ADesg mantêm-se constante para a variável Pluviometria, o que significa que o efeito principal da pluviometria não tem efeito sobre o modelo. 


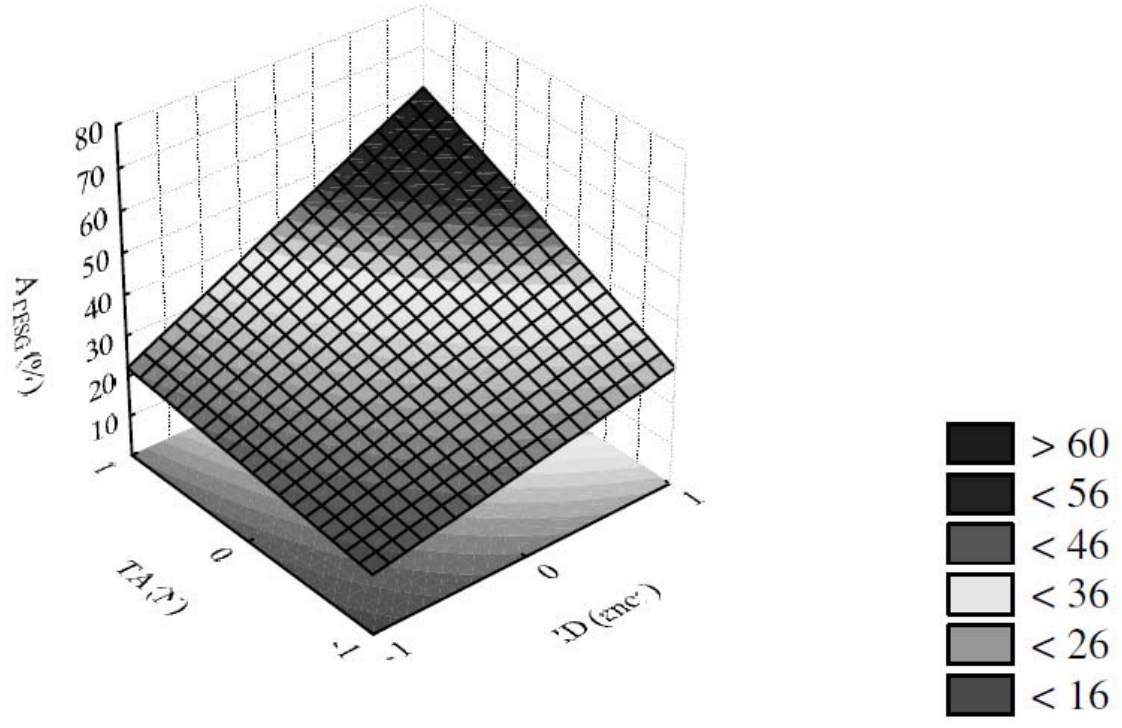

Figura 9 - Efeitos dos fatores idade e tráfego acumulado sobre o IRI

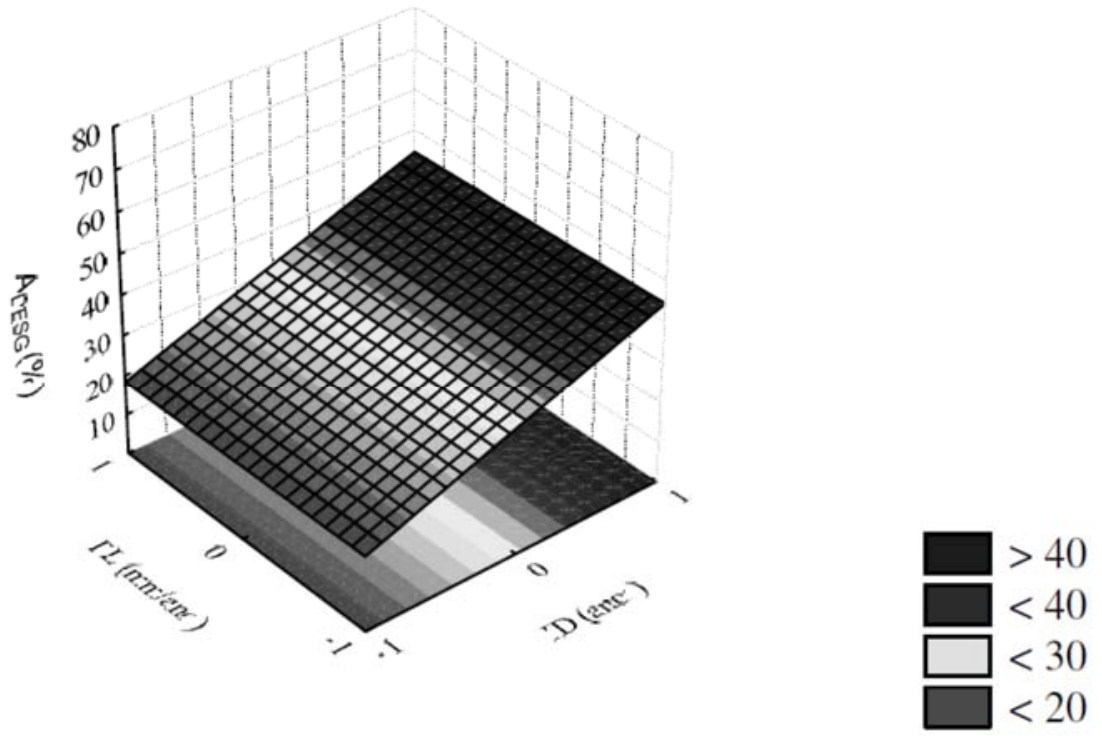

Figura 10 - Efeitos dos fatores idade e pluviometria sobre o IRI

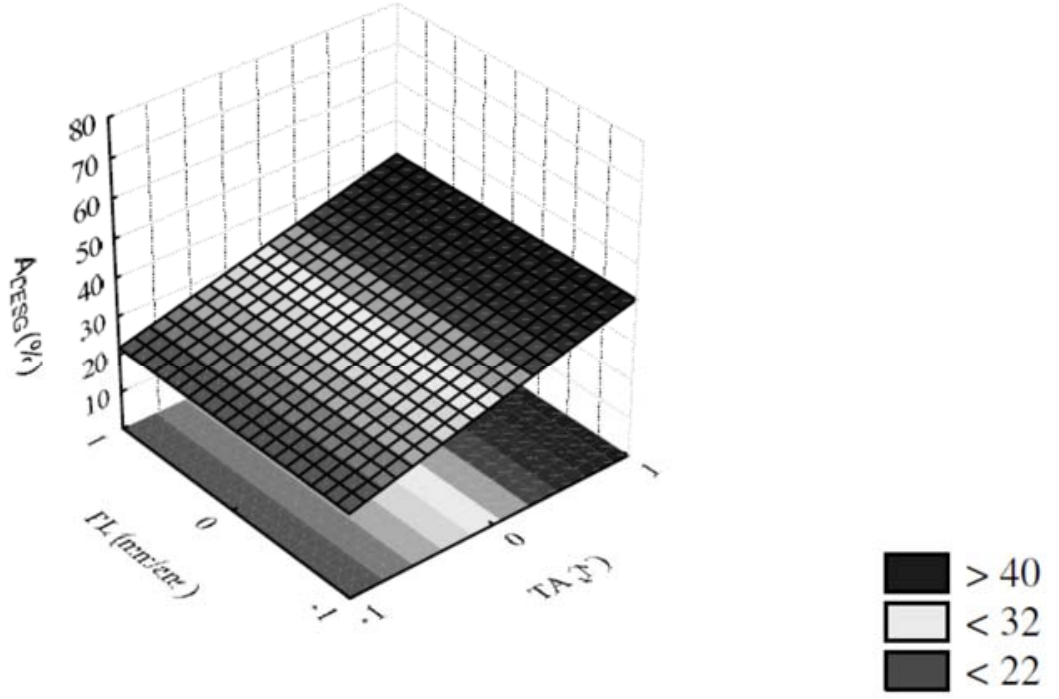

Figura 11 - Efeitos dos fatores tráfego e pluviometria sobre o IRI 


\section{CONCLUSÃO}

Foi desenvolvido um modelo de desempenho para previsão da área de desgaste considerando dados da malha rodoviária pavimentada em tratamento superficial duplo do estado da Bahia. Os resultados da ANOVA mostraram que os fatores idade, tráfego e a interação entre a idade e o tráfego apresentaram efeito significativo. O modelo desenvolvido neste trabalho apresentou ajuste aos dados observados e coeficiente de correlação $r=0,74$. Não foi possível a comparação com outros modelos de desempenho da área de desgaste superficial de pavimentos asfálticos desenvolvidos para outras redes viárias brasileiras constituídas por revestimentos em TSD, ou outro tipo de revestimento, pois não foram encontrados quando da revisão bibliográfica.

Sugere-se a comparação do modelo de desempenho desenvolvido neste trabalho com os modelos de deterioração do HDM-4, ferramenta computacional desenvolvida e utilizada pelo Banco Mundial, em planejamento e programação de investimentos e atividades de manutenção ou reabilitação de rodovias, por meio da aplicação do banco de dados do DERBA. Também, há a necessidade de estudos complementares, que incluam fatores que não puderam ser considerados neste trabalho (capacidade estrutural do pavimento, por exemplo). E, para verificação da aplicabilidade do modelo da ADesg desenvolvido, para condições específicas de outras malhas rodoviárias, podem ser utilizadas informações de bancos de dados de malhas rodoviárias de outros estados.

\section{AGRADECIMENTOS}

Ao CNPq pela concessão de uma bolsa de doutorado, ao Programa de Pós-Graduação em Engenharia de Transportes da EESC-USP, à UFBA pelo apoio aos levantamentos de campo e ao DERBA por ceder os dados para a execução desta pesquisa.

\section{REFERÊNCIAS}

American Society for Testing and Material (1996). ASTM E1777-996 (Reapproved 2002) - Standard Guide for Prioritiza- tion of Data Needs for Pavement Management. Philadelphia, USA, $09 \mathrm{p}$

Albuquerque, F. S. (2007) Sistema de gerência de pavimentos para departamentos de estradas do nordeste brasileiro. Tese (Doutorado). Universidade Federal do Rio Grande do Sul, Porto Alegre.

Benevides, S. A. S. (2006) Modelos de desempenho de pavimentos asfálticos para um sistema de gestão de rodovias estaduais do Ceará. Tese (Doutorado). COPPE, Universidade Federal do Rio de Janeiro, Rio de Janeiro.

Bennett, C. R.; Paterson, W. D. O. (2000) A guide to calibration and adaptation (Highway Development and Management HDM-4). Birmingham, United Kingtom. Highway Development and Management Series, Version 1.0, v.5, 229p.

DERBA (2005) Sugestões Técnicas para Implementação do Programa de Restauração e Manutenção Rodoviária. v. 1a 5. Departamento de Infraestrutura de Transportes da Bahia. Salvador, BA.

GEIPOT (1981) Pesquisa Sobre o Inter-relacionamento dos Custos de Construção, Conservação e Utilização de Rodovias. Empresa Brasileira de Planejamento de Transportes, Ministério dos Transportes, Brasília, DF.

Hass, R.; Hudson, W. R.; Zaniewski, J. (1994) Modern pavement management. Flórida: Krieger Publishing Company, 581 p.

Marcon, F. M. (1996) Contribuição ao desenvolvimento de um sistema de gerência de pavimentos para a malha rodoviária estadual de Santa Catarina. Tese (Doutorado). Centro Técnico aeroespacial, Instituto Tecnológico de Aeronáutica, São Paulo.

Paterson, W. D. O. (1987) Road Deterioration and Maintenance Effects: Models for Planning and Management. Published for the Word Bank, Washington, D.C.

Paterson, W. D. O. (1989) A transferable causal model for predicting roughness progression in flexible pavements. Transportation Research Record, Washington, n. 1215, p. 70-84.

Queiroz, C. A. V. (1984) Modelos de previsão de desempenho para a gerência de pavimentos no Brasil. Brasília: GEIPOT, 366 p.

Queiroz, C. A. V (1981) Performance prediction models for pavement management in Brazil. Tese (Doutorado). The University of Texas at Austin, Texas.

Soncim, S. P.; Fernandes Jr., J. L. (2012) Desenvolvimento de um modelo de desempenho de irregularidade longitudinal para a rede de rodovias em tratamento superficial duplo do estado da Bahia. Transportes, Rio de Janeiro, v. 20, n. 1, p13-21.

Yshiba, J. K. (2003) Modelos de previsão de desempenho: estudos de rodovias do estado do Paraná. Tese (Doutorado). Escola de Engenharia de São Carlos, Universidade de São Paulo, São Paulo.

Watanada, T.; Paterson, W.; Bhandi, A.; Harral, C.; Dhareshwar, A. M. e Tsunokawa, K. (1987) The Highway Design and Maintenance Standards Model. v.1. Description of de HDM III model. 